\title{
ACREDITACIÓN DE LOS POSGRADOS EN CHILE
}

\author{
Ricardo Reich*
}

* Coordinador General del Programa de Mejoramiento de la Calidad de la Educación Superior (MECESUP) del Ministerio de Educación de Chile. 


\section{RESUMEN}

En la década de los noventa, las instituciones de educación superior chilenas, preferentemente aquellas adscritas al Consejo de Rectores de Universidades, dieron inicio a un conjunto de procesos de acreditación de programas de posgrados. En la actualidad estos abarcan también las carreras de pregrado, pues la evaluación valoriza las cualidades que son necesarias para asegurar la calidad, informar adecuadamente al público y optimizar los recursos estatales y privados destinados a la educación terciaria. Conjuntamente con ello, la acreditación se ha transformado en una iniciativa que contribuye al mejoramiento de los programas de posgrado, lo que permite luego su proyección internacional. En suma, la creciente cantidad de procesos experimentales en marcha y de resoluciones de acreditación, junto al proyecto de ley para el Sistema Nacional de Acreditación, actualmente en el Congreso Nacional, auguran la instalación definitiva en Chile de un sistema de validación de la calidad de la educación superior. En este contexto, el artículo describe y cuantifica la acreditación de posgrados en Chile desde su etapa inicial guiada por la Comisión Nacional de Investigación Científica y Tecnológica hasta la actual Comisión Nacional de Acreditación de Postgrado.

\section{ABSTRACT}

In the 1990s, Chilean higher education institutions - principally the ones that are part of the Council of University Chancellors- started up a series of accreditation processes for post-graduate programs. These currently include undergraduate courses in view of the fact that evaluations assess the qualifications needed to ensure financing from the government and the private sector, because accreditation is an indicator of the fact that a given institution in particular possesses the conditions required to optimize resources. Simultaneously, accreditation has turned into a self-evaluation initiative that helps improve programs and subsequently permits their international projection. In short, the growing number of experimental processes underway and accreditation resolutions, coupled with the bill for the National Accreditation System currently being analyzed by the Chilean Congress predict that Chile will definitely have in place a system to validate the quality of higher education. In this framework, the article describes and quantifies the accreditation of postgraduate study programs in Chile, from their initial stage guided by CONICYT -the National Commission for Scientific and Technological Research-up to the current phase under the Comisión Nacional de Acreditación de Posgrados. 


\section{ACREDITACIÓN DE LOS POSGRADOS EN CHILE}

La evaluación de programas de posgrado puede ser una estrategia contemplada en la planificación y el presupuesto de la institución y de cada unidad académica. Una de las causas por las cuales la acreditación ha cobrado relevancia se debe a que la evaluación aporta mecanismos de diagnóstico y de rendición de cuentas públicas sobre las actividades y la calidad de los programas, lo cual genera un conjunto de informaciones que permite ubicarlos a nivel nacional e internacional y facilita la decisión externa sobre el financiamiento, el otorgamiento de becas, los aportes para la investigación, la cooperación internacional e incluso las donaciones.

La evaluación, como una actividad estratégica interna de las instituciones, permite que todos se beneficien de sus resultados. Las facultades y los administradores académicos logran determinar las fortalezas y debilidades de sus programas, clarificar su misión y metas futuras, tomar decisiones presupuestarias basadas en prioridades académicas; asimismo, las instituciones consiguen rendir adecuada cuenta pública a sus estudiantes, a las agencias de financiamiento y a los contribuyentes. Este diálogo interno y externo incide en que los programas mejoren en forma sostenida.

Las variables que contempla el proceso de evaluación de programas son, entre otras, la calidad de sus académicos, de sus estudiantes, el diseño curricular, las bibliotecas, los recursos educacionales, las instalaciones disponibles y la reputación que tenga el programa entre sus pares. El análisis de estas y otras variables sirve para mejorar; de forma que si se desea la superación constante, se debe planificar la revisión de manera sistemática. En la medida en que 
estos procesos formen parte habitual de la gestión institucional, las exigencias externas de acreditación serán fáciles de atender y superar.

La evaluación contribuye a la planificación de largo plazo, pues determina las prioridades institucionales y departamentales. Este proceso permite a los administradores y directivos académicos obtener información sobre el tamaño y la estabilidad de un programa. Por lo tanto, la evaluación ayuda a determinar los requerimientos futuros de planta académica de acuerdo con las proyecciones del mercado de capital humano. Esto significa, entre otras variables, fijar las necesidades de recursos informáticos, de equipamiento científico y de espacio. La evaluación establece, por lo tanto, una visión de futuro que asegura la toma de decisiones académicas y presupuestarias basadas en prioridades y no en imprecisiones o esquemas teóricos. En definitiva, la ventaja de evaluar regularmente los programas de posgrado radica en que ese proceso genera los mecanismos para un cambio eficaz con proyección en el tiempo y hacia comunidades nacionales e internacionales.

\section{LOS POSGRADOS EN CHILE: UNA MIRADA CUANTITATIVA}

Un reciente estudio realizado bajo los auspicios del Programa de Mejoramiento de la Calidad de la Educación Superior (MECESUP) ha permitido establecer a diciembre de 2002 el funcionamiento de 124 programas de doctorados (ver Cuadro $\mathrm{N}^{\circ} 1$ ): 113 de ellos en catorce universidades adscritas al Consejo de Rectores de Universidades Chilenas y once en siete universidades privadas nuevas. Estos programas consideran 1.756 estudiantes matriculados, 1.041 de ellos concentrados en la Región Metropolitana y 715 en las regiones II (6), IV (63), V (139), VII (22), VIII (317), IX (18) y X (150). La graduación fue de 92 doctores ese año.

Aunque no existe un estudio similar para la oferta de maestrías en el país, el Anuario Estadístico 2001 del Consejo de Rectores permite establecer una oferta de 437 programas de maestría (que incluyen las menciones), con una matrícula de 7.650 alumnos en veintidós universidades y una graduación de 1.473 . 


\section{Cuadro $\mathrm{N}^{\circ} 1$}

DIRECTORIO DE PROGRAMAS DE DOCTORADOS - DICIEMBRE DE 2002 A. UNIVERSIDADES DEL CONSEJO DE RECTORES

\section{UNIVERSIDAD DE CHILE}

\section{Bioquímica}

Ciencias Biomédicas

Ciencias Farmacéuticas

Ciencias Médicas

Ciencias Silvoagropecuarias y Veterinarias

Ciencias con Mención en Astronomía

Ciencias con Mención en Biología Molecular, Celular y Neurociencias

Ciencias con Mención en Computación

Ciencias con Mención en Ecología y Biología Evolutiva

Ciencias con Mención en Física

Ciencias con Mención en Geología

Ciencias con Mención en Matemática

Ciencias con Mención en Microbiología (en conjunto con la U. de Santiago de Chile)

Ciencias de la Ingeniería con Mención en Automática

Ciencias de la Ingeniería con Mención en Ciencias de los Materiales

Ciencias de la Ingeniería con Mención en Fluodinámica

Ciencias de la Ingeniería con Mención en Gestión de Operaciones

Ciencias de la Ingeniería con Mención en Ingeniería Química

Ciencias de la Ingeniería con Mención en Modelación Matemática

Derecho

Economía

Farmacología

Filosofía con Mención en Epistemología de las Ciencias Sociales

Filosofía con Mención en Ética

Filosofía con Mención en Filosofía Política

Filosofía con Mención en Literatura

Filosofía con Mención en Lingüística Hispánica

Filosofía con Mención en Metafísica

Historia con Mención en Etnohistoria

Historia con Mención en Historia de Chile

Literatura con Mención en Literatura Chilena e Hispanoamericana

Nutrición y Alimentos

Psicología

Química

Salud Pública

\section{PONTIFICIA UNIVERSIDAD CATÓLICA DE CHILE}

Ciencias Biológicas con Mención en Biología Celular y Molecular

Ciencias Biológicas con Mención en Ciencias Fisiológicas

Ciencias Biológicas con Mención en Ecología

Ciencias Biológicas con Mención en Genética Molecular y Microbiología

Ciencias de la Educación

Ciencias de la Ingeniería, Área de Ingeniería Civil

Ciencias de la Ingeniería, Área de Ciencias de la Computación

Ciencias de la Ingeniería, Área de Ingeniería Eléctrica

Ciencias de la Ingeniería, Área de Ingeniería Industrial y de Transportes

Ciencias de la Ingeniería, Área de Ingeniería Mecánica y Metalúrgica

Ciencias de la Ingeniería, Área de Ingeniería Química y Bioprocesos

Ciencias Exactas con Mención en Física

Ciencias Exactas con Mención en Matemática

Ciencias Exactas con Mención en Química 
Ciencias Médicas

Derecho

Economía

Estadística

Filología Hispánica

Filosofía

Historia

Literatura

Psicología

Teología

\section{UNIVERSIDAD DE CONCEPCIÓN}

Ciencias Ambientales

Ciencias Aplicadas con Mención en Ingeniería Matemática

Ciencias Biológicas, Área de Biología Celular y Molecular

Ciencias Biológicas, Área de Botánica

Ciencias con Mención en Química

Ciencias de la Ingeniería con Mención en Ingeniería Eléctrica

Ciencias de la Ingeniería con Mención en Ingeniería Mecánica

Ciencias de la Ingeniería con Mención en Ingeniería Química

Ciencias de la Ingeniería con Mención en Metalurgia

Ciencias Físicas

Ciencias Forestales (en conjunto con la U. Austral de Chile)

Educación

Lingüística

Literatura Latinoamericana

Oceanografía

Recursos Hídricos

UNIVERSIDAD CATÓLICA DE VALPARAÍSO

Biotecnología

Ciencias Físicas (en conjunto con la U. Técnica F. Santa María)

Ciencias con Mención en Química

Filosofía

Lingüística

Literatura

UNIVERSIDAD TÉCNICA FEDERICO SANTA MARÍA

Ciencias Físicas (en conjunto con la U. Católica de Valparaíso)

Ingeniería Electrónica

Ingeniería Mecánica

\section{UNIVERSIDAD DE SANTIAGO DE CHILE}

Ciencias con Mención en Física

Ciencias con Mención en Matemática

Ciencias con Mención en Microbiología (en conjunto con la U. de Chile)

Ciencias de la Ingeniería, Mención Automática

Ciencias de la Ingeniería, Mención Ciencia e Ingeniería de los Materiales

Ciencias de la Ingeniería, Mención en Ingeniería Informática

Ciencias de la Ingeniería, Mención en Ingeniería de Procesos

Estudios Americanos con Mención en Historia Social y Económica

Estudios Americanos con Mención en Pensamiento y Cultura

Estudios Americanos con Mención en Relaciones Internacionales

Química

\section{UNIVERSIDAD AUSTRAL DE CHILE}

Ciencias Agrarias

Ciencias Forestales (en conjunto con la U. de Concepción)

Ciencias con Mención en Biología Celular y Molecular 
Ciencias con Mención en Biología Vegetal

Ciencias con Mención en Inmunología

Ciencias con Mención en Sistemática y Ecología

Ciencias con Mención en Zoología

Ciencias Humanas con Mención en Literatura y Lingüística

Ciencias Marinas y Limnológicas

Ciencias Veterinarias

\section{UNIVERSIDAD CATÓLICA DEL NORTE}

Ciencias con Mención en Geología

\section{UNIVERSIDAD DE VALPARAÍSO}

Ciencias Empresariales

\section{UNIVERSIDAD DE LA SERENA}

Ciencias de la Educación

Desarrollo Urbano Sustentable

\section{UNIVERSIDAD DE LA FRONTERA}

Ciencias, Mención en Matemática

Ciencias de Recursos Naturales

\section{UNIVERSIDAD DE MAGALLANES}

Energías Renovables

\section{UNIVERSIDAD DE TALCA}

Ciencias con Mención en Ingeniería Genética Vegetal

Ciencias con Mención en Investigación y Desarrollo de Productos Naturales

\section{UNIVERSIDAD DE LOS LAGOS}

Ciencias de la Educación

Educación Física

\section{B. UNIVERSIDADES PRIVADAS}

UNIVERSIDAD ACADEMIA DE HUMANISMO CRISTIANO

Educación

UNIVERSIDAD AUTÓNOMA DEL SUR

Administración

Educación

UNIVERSIDAD CENTRAL DE CHILE

Arquitectura y Patrimonio Cultural-Ambiental

UNIVERSIDAD DE ARTES Y CIENCIAS SOCIALES

Estudio de las Sociedades Latinoamericanas

UNIVERSIDAD DE LOS ANDES

Derecho

UNIVERSIDAD DIEGO PORTALES

Comunicación, Derecho a la Información y Ética en España y Latinoamérica

\section{UNIVERSIDAD NACIONAL ANDRÉS BELLO}

Biociencias Moleculares

Biotecnología

Fisicoquímica Molecular

Psicología con Mención en Psicoanálisis 


\section{LA ACREDITACIÓN EN CHILE: LA ETAPA INICIAL EN CONICYT}

La acreditación de programas de posgrado en Chile comienza el año 1991, cuando la Comisión Nacional de Investigación Científica y Tecnológica (CONICYT) implementó procesos de aseguramiento de calidad con el propósito de resguardar niveles mínimos de calidad académica en aquellos centros universitarios que recibían, a través de la captación de becarios, recursos públicos. El proceso se aplicó a programas vigentes de maestría y doctorado ofrecidos en el país, en razón a solicitudes de las instituciones de las cuales dependían. Conceptualmente, se diseñó como una evaluación externa, donde los antecedentes aportados por los programas -en formularios ad hoceran evaluados por comités técnicos en áreas disciplinarias integradas por académicos destacados de la comunidad científica nacional.

CONICYT invitó para estos procesos de acreditación a científicos de diferentes ámbitos del conocimiento con el fin de que integraran comisiones técnicas cuyos propósitos eran evaluar los programas de acuerdo a criterios generales preestablecidos y específicos de la disciplina correspondiente. Los objetivos principales de esta fase inicial de acreditación fueron: propiciar la consolidación del sistema de posgrados chilenos, adoptando criterios de calidad reconocidos internacionalmente; promover la formación de recursos humanos altamente calificados; propiciar una oferta más racional de programas y dar a conocer información confiable acerca de la calidad de la oferta educativa a la comunidad. El período de validez de la acreditación se limitó a cinco años, estableciéndose como principios de operación la transparencia y la confiabilidad de los procedimientos empleados cuando ellos toman en consideración los estándares internacionales y los objetivos institucionales.

Durante este primer período, CONICYT convocó a procesos de acreditación de maestrías y doctorados los años 1990, 1993, 1994 y 1996. Estos fueron respondidos por catorce universidades para 58 programas de doctorado -que incluyen 25 especialidades- y 176 programas de maestría con numerosas especialidades. Considerando el total de procesos de acreditación sometidos a trámite, para el período 1990-1996, CONICYT resolvió favorablemente la acreditación de 186 programas de posgrado, no otorgando la acreditación a otros 94 . 
La acreditación de posgrados por CONICYT, más los procesos de licenciamiento de nuevas universidades desarrollados por el Consejo Superior de Educación y los sistemas de autoevaluación iniciados por las universidades de Concepción y de Chile, sentaron las bases de la autorregulación universitaria en Chile. Aunque CONICYT se orientó principalmente a la evaluación de la calidad de los postulantes a becas nacionales de posgrado, su aporte fue meritorio ya que, por su seriedad, permitió introducir la cultura de la evaluación.

\section{LA ACREDITACIÓN EN CHILE: LA ETAPA DE LA CONAP}

Durante el año 1999, el ministerio de Educación inició el Programa MECESUP cuyo financiamiento proviene de un préstamo del Banco Internacional de Reconstrucción y Fomento (4404-CH) y recursos del Gobierno de Chile. Este programa considera como un componente importante el aseguramiento de la calidad de la educación superior, especialmente en lo que respecta a la formación de profesionales de título y de posgrado en las áreas de maestría y de doctorado. Por lo tanto, desde el objetivo inicial de CONICYT respecto de la asignación de becas nacionales, se amplía a propósitos más generales, a saber, la evaluación y el mejoramiento de los programas junto a una difusión de la oferta educacional. Como a esa fecha no existían cuerpos legales que definieran y precisaran la acreditación en el país, se opta en el Ministerio de Educación por la implementación transitoria de dos comisiones nacionales de acreditación: la Comisión Nacional de Acreditación de Pregrados (CNAP, Decreto N 55 de 1999) y la Comisión Nacional de Acreditación de Postgrados (CONAP, Decretos $N^{\circ} 225,3380$ y 184 de 1999, 2000 y 2002). Ambas comisiones están dotadas de una adecuada autonomía, amplias facultades y múltiples recursos para operar lo que les ha permitido generar las bases de un Sistema Nacional de Acreditación (SINAC). Este fue presentado como proyecto de ley por el Supremo Gobierno al Congreso Nacional a fines del año 2002. La CNAP y la CONAP también tienen entre sus funciones colaborar con el Fondo Competitivo del Programa MECESUP, acreditando las becas de posgrado asignadas. Indirectamente, estas comisiones también intervienen en la elegibilidad de las instituciones para efectos de obtener los recursos disponibles de apoyo a los posgrados nacionales. 
La CONAP está construida sobre la base de la experiencia adquirida por CONICYT, lo que ha permitido optimizar sus procedimientos, avanzar con rapidez y explorar nuevos modelos de acreditación, especialmente para las maestrías. Para los programas que otorgan ese grado, se ha diseñado e implementado un modelo de acreditación basado en procesos autoevaluativos con auditoría académica externa. Con respecto a los doctorados, la CONAP a partir del año 2000 elaboró las bases de un nuevo ciclo de acreditación. Este tenía por objetivo emitir una opinión técnica sobre la calidad de los programas sometidos a análisis, basando el juicio en los informes de autoevaluación -generados a partir de criterios estándares de calidad-, visitas a terreno y el juicio de pares académicos externos nacionales o internacionales.

\section{ACREDITACIÓN DE DOCTORADOS}

Como modelo de acreditación, la CONAP ha seguido utilizando para los programas de doctorado la evaluación externa a través de comités de evaluación por áreas de especialidad (actualmente dieciséis) y visitas, en casos seleccionados, a la comunidad académica y a sus instalaciones. Estos comités, integrados por académicos destacados de universidades chilenas, evalúan los antecedentes aportados por los programas solicitantes; a saber, información sobre el personal académico, su formación de posgrado, gestión en investigación y productividad medida por publicaciones de calidad y proyección internacional, planes de estudio, requisitos de admisión y graduación, infraestructura disponible y capacidad de gestión y administración, como también antecedentes de evaluaciones realizadas con anterioridad.

Los programas son acreditados por distintos períodos, de acuerdo con su nivel de calidad. La acreditación por seis años está reservada a programas de doctorado que hayan sido acreditados en forma continua durante los dos últimos períodos de evaluación de CONICYT y que demuestren continuidad de su calidad a través de la entrega de datos complementarios. La acreditación por cuatro años es para programas nuevos sin objeciones del respectivo comité de evaluadores de área y programas acreditados previamente con observaciones menores, incluyendo menciones. La acreditación por dos años es para programas 
nuevos con observaciones significativas, incluyendo el mejoramiento de sus cuadros académicos. Para efectos de información pública de los resultados de acreditación, se ha optado por dar a conocer, como consecuencia de los procesos, exclusivamente los acreditados. Los procesos de doctorado sancionados por la CONAP hasta esta fecha son informados en el Cuadro $\mathrm{N}^{\circ} 2$.

\section{Cuadro $\mathrm{N}^{\circ} 2$}

\section{PROGRAMAS DE DOCTORADO ACREDITADOS}

(CONAP 20/03/2003)

\begin{tabular}{|c|c|c|c|c|}
\hline UNIVERSIDAD/DOCTORADO & RESOLUCIÓN & AÑOS & ACREDITA & CIÓN \\
\hline UNIVERSIDAD DE CHILE & & & & \\
\hline Doctorado en Ciencias Mención/Ecología y Biología Evolutiva & ACREDITADO & 2 años & octubre & 2000 \\
\hline Doctorado en Bioquímica & ACREDITADO & 2 años & octubre & 2000 \\
\hline Doctorado en Ciencias M/Biología Molecular, Celular y Neurociencias & ACREDITADO & 6 años & octubre & 2000 \\
\hline Doctorado en Ciencias M/Microbiología (conjunto con USACH) & RE-ACREDITADO & 6 años & marzo & 2003 \\
\hline Doctorado en Ciencias Biomédicas & ACREDITADO & 2 años & octubre & 2000 \\
\hline Doctorado en Ciencias Médicas & ACREDITADO & 2 años & septiembre & 2001 \\
\hline Doctorado en Ciencias de la Ingeniería M/Ciencia de los Materiales & RE-ACREDITADO & 4 años & diciembre & 2002 \\
\hline Doctorado en Ciencias de la Ingeniería M/Modelación Matemática & ACREDITADO & 6 años & octubre & 2000 \\
\hline Doctorado en Ciencias de la Ingeniería Mención Química & ACREDITADO & 4 años & octubre & 2000 \\
\hline Doctorado en Ciencias Mención/Física & ACREDITADO & 4 años & octubre & 2000 \\
\hline Doctorado en Ciencias Mención/Astronomía & RE-ACREDITADO & 4 años & marzo & 2003 \\
\hline Doctorado en Química & ACREDITADO & 6 años & octubre & 2000 \\
\hline Doctorado en Ciencias Mención/Matemática & ACREDITADO & 2 años & octubre & 2000 \\
\hline Doctorado en Ciencias Mención/Geología & RE-ACREDITADO & 4 años & marzo & 2003 \\
\hline Doctorado en Historia Mención/Etnohistoria & RE-ACREDITADO & 2 años & diciembre & 2002 \\
\hline Doctorado en Historia Mención/Historia de Chile & RE-ACREDITADO & 2 años & diciembre & 2002 \\
\hline Doctorado en Filosofía Mención/Filosofía Política & ACREDITADO & 2 años & octubre & 2000 \\
\hline Doctorado en Filosofía Mención/Etica & ACREDITADO & 2 años & octubre & 2000 \\
\hline Doctorado en Filosofía M/Epistemología de las Ciencias Sociales & ACREDITADO & 2 años & octubre & 2000 \\
\hline Doctorado en Filosofía Mención/Metafísica & ACREDITADO & 2 años & octubre & 2000 \\
\hline Doctorado en Literatura M/Literatura Chilena e Hispanoamericana & ACREDITADO & 4 años & octubre & 2000 \\
\hline Doctorado en Economía & ACREDITADO & 4 años & octubre & 2000 \\
\hline Doctorado en Derecho & ACREDITADO & 2 años & marzo & 2002 \\
\hline Doctorado en Nutrición y Alimentos & ACREDITADO & 2 años & julio & 2002 \\
\hline Doctorado en Cs. Silvoagropecuarias y Veterinarias & ACREDITADO & 2 años & julio & 2002 \\
\hline Doctorado en Salud Pública & ACREDITADO & 2 años & octubre & 2002 \\
\hline
\end{tabular}

\begin{tabular}{|c|c|c|c|c|}
\hline PONTIFICIA UNIVERSIDAD CATOLICA DE CHILE & & & & \\
\hline Doctorado en Ciencias Biológicas Mención/Ecología & ACREDITADO & 6 años & octubre & 2000 \\
\hline Doctorado en Ciencias Biológicas M/Biología Celular y Molecular & ACREDITADO & 6 años & octubre & 2000 \\
\hline Doctorado en Ciencias Biológicas M/Ciencias Fisiológicas & ACREDITADO & 6 años & octubre & 2000 \\
\hline Doctorado en Ciencias Biológicas M/Genética Molecular y Microbiología & ACREDITADO & 2 años & octubre & 2000 \\
\hline Doctorado en Ciencias Médicas & ACREDITADO & 2 años & octubre & 2000 \\
\hline $\begin{array}{l}\text { Doctorado en Ciencias de la Ingeniería, Área de Especialización } \\
\text { Ingeniería Química y Bioprocesos }\end{array}$ & ACREDITADO & 4 años & octubre & 2000 \\
\hline $\begin{array}{l}\text { Doctorado en Ciencias de la Ingeniería, Área de Especialización } \\
\text { Ciencia de la Computación }\end{array}$ & ACREDITADO & 2 años & octubre & 2000 \\
\hline $\begin{array}{l}\text { Doctorado en Ciencias de la Ingeniería, Área de Especialización } \\
\text { Ingeniería Industrial y de Transporte }\end{array}$ & ACREDITADO & 2 años & octubre & 2000 \\
\hline
\end{tabular}


Doctorado en Ciencias de la Ingeniería, Área de Especialización Ingeniería Eléctrica

Doctorado en Ciencias de la Ingeniería, Área de Especialización

Ingeniería Civil

Doctorado en Ciencias Exactas Mención/Física

Doctorado en Ciencias Exactas Mención/Química

Doctorado en Ciencias Exactas Mención/Matemática

Doctorado en Estadística

Doctorado en Historia

Doctorado en Filosofía

Doctorado en Psicología

Doctorado en Ciencias de la Educación

Doctorado en Teología

\begin{tabular}{|l|l|l|l|}
\hline ACREDITADO & 4 años & octubre & 2000 \\
\hline ACREDITADO & 2 años & junio & 2002 \\
\hline ACREDITADO & 4 años & octubre & 2000 \\
\hline ACREDITADO & 6 años & octubre & 2000 \\
\hline ACREDITADO & 2 años & octubre & 2000 \\
\hline ACREDITADO & 2 años & octubre & 2000 \\
\hline ACREDITADO & 4 años & octubre & 2000 \\
\hline ACREDITADO & 4 años & octubre & 2000 \\
\hline ACREDITADO & 2 años & octubre & 2000 \\
\hline ACREDITADO & 2 años & octubre & 2000 \\
\hline ACREDITADO & 2 años & julio & 2002 \\
\hline
\end{tabular}

\section{UNIVERSIDAD DE SANTIAGO DE CHILE}

Doctorado en Ciencias m/Física

\begin{tabular}{|l|l|lr|}
\hline & & \multicolumn{2}{|c|}{} \\
\hline ACREDITADO & 2 años & noviembre & 2000 \\
\hline ACREDITADO & 6 años & octubre & 2000 \\
\hline ACREDITADO & 2 años & octubre & 2000 \\
\hline ACREDITADO & 2 años & octubre & 2000 \\
\hline ACREDITADO & 2 años & octubre & 2000 \\
\hline ACREDITADO & 2 años & julio & 2001 \\
\hline
\end{tabular}

Doctorado en Química

Doctorado en Ciencias m/Microbiología (conjunto con UCH)

Doctorado en Estudios Americanos m/Historia Social y Económica

Doctorado en Estudios Americanos m/Pensamiento y Cultura

$S$ ACREDITADO

2 años julio

2001

\section{UNIVERSIDAD DE CONCEPCION}

Doctorado en Ciencias Biológicas, Área de Especialización Botánica Doctorado en Ciencias Biológicas, Área de especialización Biología Celular y Molecular

Doctorado en Ciencias Aplicadas m/Ingeniería Matemática

Doctorado en Ciencias de la Ingeniería m/Ingeniería Química

Doctorado en Ciencias de la Ingeniería m/Ingeniería Eléctrica

Doctorado en Ciencias Mención/Química

Doctorado en Ciencias Físicas

Doctorado en Oceanografía

Doctorado en Lingüística

Doctorado en Literatura Latinoamericana

Doctorado en Ciencias Forestales (UCO/UACH)

\begin{tabular}{|l|l|ll}
\hline & & & \\
\hline ACREDITADO & 4 años & octubre & 2000 \\
\hline ACREDITADO & 2 años & octubre & 2000 \\
\hline ACREDITADO & 4 años & octubre & 2000 \\
\hline RE-ACREDITADO & 6 años & diciembre & 2002 \\
\hline RE-ACREDITADO & 2 años & diciembre & 2002 \\
\hline ACREDITADO & 6 años & octubre & 2000 \\
\hline ACREDITADO & 2 años & diciembre & 2001 \\
\hline RE-ACREDITADO & 6 años & diciembre & 2002 \\
\hline RE-ACREDITADO & 2 años & diciembre & 2002 \\
\hline RE-ACREDITADO & 2 años & diciembre & 2002 \\
\hline ACREDITADO & 2 años & julio & 2002 \\
\hline
\end{tabular}

\section{UNIVERSIDAD AUSTRAL DE CHILE}

\begin{tabular}{ll|l|l}
\hline Doctorado en Ciencias m/Sistemática y Ecología & ACREDITADO & 2 años & octubre 2000
\end{tabular}

\begin{tabular}{ll|l|l}
\hline Doctorado en Ciencias m/Biología Celular y Molecular & ACREDITADO & 6 años octubre 2000
\end{tabular}

\begin{tabular}{|l|l|l|l}
\hline Doctorado en Ciencias Veterinarias & ACREDITADO & 2 años octubre 2000
\end{tabular}

\begin{tabular}{ll|l|l}
\hline Doctorado en Ciencias Forestales (UCO/UACH) & ACREDITADO & 2 años julio 2002
\end{tabular}

UNIVERSIDAD TÉCNICA FEDERICO SANTA MARÍA

Doctorado en Ciencias Físicas UTFSM/UCV
RE-ACREDITADO 6 años diciembre 2002
UNIVERSIDAD CATOLICA DE VALPARAISO

Doctorado en Ciencias Mención/Química

Doctorado en Lingüística

ACREDITADO 6 años octubre 2000

\begin{tabular}{l|l|l|}
\hline ACREDITADO & 6 años & septiembre 2001
\end{tabular}




\section{ACREDITACIÓN DE MAESTRÍAS}

Aunque en el caso de las maestrías, la CONAP sigue ofreciendo la modalidad de acreditación por evaluación externa, resolvió en el año 2001 experimentar con un nuevo modelo que fomenta la autorregulación institucional de estos programas. Este cambio se debe al interés de fomentar las capacidades institucionales que aseguran la calidad y no sólo recurrir a la evaluación externa para obtener una adecuada información que sitúe al programa con respecto a la oferta educacional.

Este nuevo procedimiento de acreditación, de tipo experimental, ha sido acogido y seleccionado mayoritariamente por las universidades del Consejo de Rectores y se encuentra en plena fase de implementación. Se trata en este caso de una opción que las universidades han tomado responsablemente y en la que comprometen esfuerzos internos significativos, conscientes de que la violación de su marco reglamentario puede significar el término de su participación y una vuelta a la evaluación externa tradicional. En concreto, significa que las instituciones deben priorizar qué programas solicitan acreditar luego de una fase de autoevaluación completa. Este programa de revisión contempla la realización de un proceso participativo de diagnóstico estratégico, la generación de un informe autoevaluativo, la verificación con pares externos (al menos uno de ellos debe ser aprobado por la CONAP), el informe final de autoevaluación (que integra ambas experiencias) y un plan de acción de cambios para el mejoramiento del programa. Solo después de ello, la CONAP contrata un auditor académico independiente, quien analiza toda la información disponible para el proceso de autorrevisión del programa de maestría, realiza una visita en terreno con el fin de determinar las capacidades de autoevaluación institucionales y la calidad del proceso e informa. Este informe será determinante para la resolución de acreditación de la CONAP. Se trata, por lo tanto, de un modelo metaevaluativo. La autoevaluación se realiza separadamente para las menciones de una determinada especialidad.

Se informan públicamente los resultados de la acreditación de maestrías, señalándose solo aquellas que calificaron positivamente, tal como sucede con los doctorados. Asimismo, los programas son 
acreditados por distintos períodos, de acuerdo con su nivel de calidad. La acreditación por cinco años está reservada a programas que hayan sido evaluados por más de un período; por cuatro años, para programas nuevos sometidos a autoevaluación con auditoría académica externa o antiguos programas que hayan obtenido observaciones importantes; y por dos años, para programas nuevos o antiguos con observaciones significativas. Los procesos de maestría sancionados por la CONAP hasta esta fecha son informados en el Cuadro $\mathrm{N}^{\circ} 3$.

\author{
Cuadro $\mathrm{N}^{\circ} 3$ \\ PROGRAMAS DE MAESTRIA ACREDITADOS \\ (CONAP 20/03/2003)
}

\begin{tabular}{l|l|l|l|l|}
\cline { 2 - 5 } UNIVERSIDAD/MAESTRÍA & RESOLUCIÓN & AÑOS & ACREDITACIÓN \\
\hline UNIVERSIDAD DE CHILE & & & & \\
\hline Magister en Estudios Latinoamericanos & RE-ACREDITADO & 2 años & diciembre & 2002 \\
\hline Magister en Estudios de Género y Cultura menciones: Cs. Sociales; & & & & \\
Humanidades & RE-ACREDITADO & 2 años & diciembre & 2002 \\
\hline Magister en Economía Aplicada & ACREDITADO & 4 años & octubre & 2001 \\
\hline Magister en Gestión y Políticas Públicas & ACREDITADO & 4 años & octubre & 2001 \\
\hline Magister en Gestión de Operaciones & ACREDITADO & 4 años & octubre & 2001 \\
\hline Magister en Gestión y Dirección de Empresas & ACREDITADO & 4 años & octubre & 2001 \\
\hline Magister en Ciencias Mención/Geología & ACREDITADO & 4 años & diciembre & 2001 \\
\hline Magister en Ciencias de la Ing. m/Transportes & ACREDITADO & 4 años & marzo & 2002 \\
\hline
\end{tabular}

\begin{tabular}{|c|c|c|c|c|}
\hline \multicolumn{5}{|l|}{ PONTIFICIA UNIVERSIDAD CATÓLICA } \\
\hline Magister en Psicología mención Psicología Educacional & ACREDITADO & 4 años & octubre & 2002 \\
\hline Magister en Psicología mención Psicología Clínica & ACREDITADO & 4 años & octubre & 2002 \\
\hline Magister en Psicología mención Psicología Social Comunitaria & ACREDITADO & 4 años & octubre & 2002 \\
\hline Magister en Letras mención Literatura & ACREDITADO & 4 años & octubre & 2002 \\
\hline Magister en Letras mención Lingüística & ACREDITADO & 4 años & octubre & 2002 \\
\hline Magister en Ciencias Vegetales & ACREDITADO & 4 años & octubre & 2002 \\
\hline Magister en Economía Agraria & ACREDITADO & 4 años & octubre & 2002 \\
\hline Magister en Ciencias Animales & ACREDITADO & 4 años & octubre & 2002 \\
\hline Magister en Arquitectura & ACREDITADO & 2 años & octubre & 2002 \\
\hline Magister en Asentamientos Humanos y Medio Ambiente & ACREDITADO & 2 años & octubre & 2002 \\
\hline Magister en Desarrollo Urbano & ACREDITADO & 2 años & octubre & 2002 \\
\hline Magister en Ciencias de la Ingeniería m/Ing. Mecánica y Metalúrgica & ACREDITADO & 4 años & octubre & 2002 \\
\hline $\begin{array}{l}\text { Magister en Ciencias de la Ingeniería mención Ingeniería Estructural } \\
\text { y Geotécnica }\end{array}$ & ACREDITADO & 4 años & octubre & 2002 \\
\hline Magister en Ciencias de la Ingeniería m/Ing. Hidráulica y Ambiental & ACREDITADO & 4 años & octubre & 2002 \\
\hline Magister en Ciencia de la Ingeniería m/Ing. y Gestión de Construcción & ACREDITADO & 4 años & octubre & 2002 \\
\hline Magister en Ciencias de la Ingeniería m/Ing. Química y Bioprocesos & ACREDITADO & 4 años & octubre & 2002 \\
\hline Magister en Ciencias de la Ingeniería m/Ciencias de la Computación & ACREDITADO & 4 años & octubre & 2002 \\
\hline Magister en Ciencias de la Ingeniería $\mathrm{m} / \mathrm{Ing}$. de Transporte & ACREDITADO & 4 años & octubre & 2002 \\
\hline Magister en Ciencias de la Ingeniería m/Ing. Eléctrica & ACREDITADO & 4 años & octubre & 2002 \\
\hline Magister en Ciencias de la Ingeniería Mención/Minería & ACREDITADO & 2 años & octubre & 2002 \\
\hline Magister en Ciencias de la Ingeniería $\mathrm{m} / \mathrm{Ing}$. Industrial y de Sistemas & ACREDITADO & 4 años & octubre & 2002 \\
\hline Magister en Ciencias Exactas Mención/Matemáticas & ACREDITADO & 4 años & octubre & 2002 \\
\hline Magister en Ciencias Exactas Mención/Física & ACREDITADO & 4 años & octubre & 2002 \\
\hline Magister en Estadística & ACREDITADO & 4 años & octubre & 2002 \\
\hline Magister en Nutrición & ACREDITADO & 4 años & octubre & 2002 \\
\hline
\end{tabular}




\section{UNIVERSIDAD/MAESTRÍA}

Magister en Sociologia

Magister en Ciencias de la Educación m/Administración Educacional

Magister en Ciencias de la Educación m/Diseño Instruccional

Magister en Ciencias de la Educación m/Educación Inicial

Magister en Ciencias de la Educación m/Currículum

Magister en Ciencias de la Educación m/Dificultades del Aprendizaje

Magister en Ciencias de la Educación m/Evaluación Educacional

Magister en Salud y Humanización

Magister en Teología
RESOLUCION ANOS ACREDITACION

ACREDITADO 2 años octubre 2002

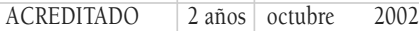

ACREDITADO 2 años octubre 2002

ACREDITADO 2 años octubre 2002

ACREDITADO 2 años octubre 2002

ACREDITADO 2 años octubre 2002

ACREDITADO 2 años octubre 2002

ACREDITADO 2 años octubre 2002

ACREDITADO 2 años octubre 2002

UNIVERSIDAD DE SANTIAGO DE CHILE

Magister en Literatura Hispanoamericana

\begin{tabular}{l|l|l|} 
ACREDITADO & 2 años & abril \\
\end{tabular}

ACADEMIA DE GUERRA DEL EJERCITO

Magister en Ciencias Militares ACREDITADO 4 años agosto

UNIVERSIDAD CATOLICA DEL NORTE

Magister en Ciencias del Mar

Magister en Antropología (UCN/UTARAPACA)

ACREDITADO 2 años noviembre 2000

ACREDITADO 2 años julio

2002

UNIVERSIDAD DE ANTOFAGASTA

\begin{tabular}{|l|l|l|l|} 
Magister en Ciencias de la Ingeniería m/Ing. de Procesos Minerales & ACREDITADO & 2 años junio 2002
\end{tabular}

\section{UNIVERSIDAD DE CONCEPCION}

Magister en Ciencias m/Botánica

Magister en Ciencias Mención/Zoología

Magister en Ciencias Mención/Microbiología

Magister en Ciencias de la Computación

Magister en Economía de Recursos Naturales y Medio Ambiente

Magister en Enfermería

Magister en Ingeniería Agrícola

Magister en Ciencias Farmacéuticas

Magister en Historia

Magister en Ciencias Mención/Matemáticas

Magister en Cs. de la Ingeniería m/Ing. Eléctrica

Magister en Literatura Hispánica

Magister en Ciencias Mención/Oceanografía

\begin{tabular}{|l|l|l|l|}
\hline & & & \\
\hline ACREDITADO & 4 años & junio & 2002 \\
\hline ACREDITADO & 4 años & junio & 2002 \\
\hline ACREDITADO & 4 años & junio & 2002 \\
\hline ACREDITADO & 4 años & junio & 2002 \\
\hline ACREDITADO & 4 años & junio & 2002 \\
\hline ACREDITADO & 2 años & junio & 2002 \\
\hline ACREDITADO & 2 años & junio & 2002 \\
\hline ACREDITADO & 2 años & agosto & 2002 \\
\hline ACREDITADO & 2 años & agosto & 2002 \\
\hline ACREDITADO & 2 años & agosto & 2002 \\
\hline ACREDITADO & 4 años & agosto & 2002 \\
\hline ACREDITADO & 4 años & agosto & 2002 \\
\hline ACREDITADO & 4 años & agosto & 2002 \\
\hline
\end{tabular}

\section{UNIVERSIDAD AUSTRAL DE CHILE}

Magister en Ciencias mención Recursos Forestales

Magister en Ciencias Mención/Salud Animal

Magister en Ciencias Mención/Producción Animal

Magister en Ciencia y Tecnología de la Leche

Magister en Ciencias Vegetales Mención/Fisiología Vegetal

Magister en Ciencias Vegetales Mención/Mejoramiento Vegetal

Magister en Ciencias Vegetales Mención/Producción Vegetal

Magister en Ciencias Vegetales Mención/Protección Vegetal

Magister en Administración de Empresas

Magister en Desarrollo Rural

Magister en Comunicación

Magister en Microbiología

Magister en Economía y Gestión Regional

\begin{tabular}{|l|l|l|l|}
\hline ACREDITADO & 2 años & noviembre & 2000 \\
ACREDITADO & 4 años & enero & 2002 \\
\hline ACREDITADO & 4 años & enero & 2002 \\
\hline ACREDITADO & 2 años & enero & 2002 \\
\hline ACREDITADO & 4 años & enero & 2002 \\
\hline ACREDITADO & 4 años & enero & 2002 \\
\hline ACREDITADO & 4 años & enero & 2002 \\
\hline ACREDITADO & 4 años & enero & 2002 \\
\hline ACREDITADO & 2 años & enero & 2002 \\
\hline ACREDITADO & 2 años & enero & 2002 \\
\hline ACREDITADO & 2 años & enero & 2002 \\
\hline ACREDITADO & 4 años & enero & 2002 \\
\hline ACREDITADO & 2 años & junio & 2002 \\
\hline
\end{tabular}




\begin{tabular}{|c|c|c|c|}
\hline UNIVERSIDAD/MAGISTER & RESOLUCIÓN & AÑOS & ACREDITACIÓN \\
\hline UNIVERSIDAD DE LOS LAGOS & & & \\
\hline $\begin{array}{l}\text { Magister en Ciencias mención Producción, Manejo y Conservación } \\
\text { de Recursos Naturales }\end{array}$ & ACREDITADO & 2 años & noviembre 2000 \\
\hline
\end{tabular}

UNIVERSIDAD CATOLICA DE VALPARAÍSO

\begin{tabular}{|l|l|l|l|}
\hline Magister en Ciencias de la Ingeniería m/Ing. Bioquímica & ACREDITADO & 2 años & octubre 2002 \\
\hline
\end{tabular}

\begin{tabular}{l|l|l|l|}
\hline Magister en Lingüística Aplicada & ACREDITADO & 2años & octubre 2002
\end{tabular}

\section{CONSECUENCIAS DE LA ACREDITACIÓN DE POSGRADOS}

La acreditación de programas de posgrado en Chile favorece la generación de capacidades de autorregulación de las universidades que la contemplan como una estrategia de mejoramiento académico. Dado que la acreditación permite una progresión continua de la calidad, el número de procesos ha ido creciendo sostenidamente, desde 39 (en 1998) hasta 72 en la actualidad.

Por otro lado, la acreditación impacta también sobre la selección de los postulantes al posgrado que requieren financiamiento para sus estudios a través de becas nacionales (por ejemplo, CONICYT, Programa MECESUP e Iniciativa Milenio) y sobre la elegibilidad a recursos del Estado para su fomento y desarrollo. Este es el caso del Programa MECESUP que, a través de su Fondo Competitivo, financia el apoyo de capital humano e infraestructura a programas de posgrado ya acreditados o nuevos programas estratégicos para el desarrollo del país. La pérdida de la acreditación para un programa de posgrado significa la dura sanción de perder el acceso a becas y a recursos de fomento del Estado.

Otro efecto positivo de la exigencia de la acreditación o del licenciamiento de la CONAP para programas de posgrado nuevos es la asignación de recursos públicos. Este ingreso de recursos trae importantes beneficios para la gestión más efectiva de los programas. 
Por un lado, permite incentivar el mejoramiento de la calidad desde el punto de vista corporativo, con un diseño de programas nuevos de forma más responsable y pertinente, pues se piensa en las características y alcances que debiera poseer para sortear exitosamente su licenciamiento. En el caso de una pérdida de la acreditación, la institución afectada deberá tomar decisiones de rediseño. Para esto es vital que el programa sancionado cuente con los aportes institucionales que aseguren su recuperación, consolidación y reacreditación.

Mirado el valor de la acreditación desde el punto de vista del Estado o del sector privado, esta evaluación permite asegurar una más eficiente y efectiva asignación de recursos, apoyando así a iniciativas que cuentan con un adecuado aseguramiento de calidad o a proyectos que poseen el respaldo y las garantías corporativas apropiadas.

\section{CONCLUSIONES}

En la década de los noventa, las instituciones de educación superior chilenas, preferentemente adscritas al Consejo de Rectores de Universidades, han visto el inicio y la progresiva implementación de procesos de acreditación de programas, primero de posgrados en CONICYT y más adelante de carreras en la CNAP y de posgrados de maestría y doctorado en la CONAP. Esta evaluación ha permitido contar con registros públicos sobre la calidad de la oferta educacional universitaria. En el caso de los posgrados, además, ha servido como una estrategia para lograr el mejoramiento de los programas y su proyección internacional. La creciente cantidad de procesos experimentales en marcha, junto al proyecto de ley para el Sistema Nacional de Acreditación actualmente en el Congreso Nacional, auguran la instalación definitiva en Chile de un sistema de aseguramiento de la calidad de la educación superior y, en particular, de los posgrados, moderno y efectivo. 
